\title{
Multiple Dural Arteriovenous Fistulas in the Superior Sagittal Sinus Region with Reversible Parkinsonism and Urinary Incontinence as Rare Presentations: A Case Report
}

\author{
Yu $\mathrm{W}^{1}$, Huang $\mathrm{Y}^{2}, \mathrm{Wu} \mathrm{J}^{1}, \mathrm{Wu} \mathrm{J}^{1}, \mathrm{Li}^{1}{ }^{1}$ and $\mathrm{J}$ in $\mathrm{H}^{2 *}$ \\ ${ }^{1}$ Department of Neurology, Peking University Shenzhen \\ Hospital, China \\ ${ }^{2}$ Department of Neurology, Peking University First \\ Hospital, China \\ *Corresponding author: Haiqiang J in, Department \\ of Neurology, Peking University First Hospital, 8 Xishiku \\ Street Xicheng District, Beijing, China
}

Received: August 27, 2021; Accepted: September 29, 2021; Published: October 06, 2021

\begin{abstract}
Background: Dural Arteriovenous Fistula (DAVF), characterized by abnormally direct connections between meningeal arteries and meningeal veins or dural venous sinuses, is a rare subtype among the intracranial arteriovenous malformations. Although the clinical manifestations of DAVF are very variable depending on the locations and size of venous drainage, parkinsonism and urinary incontinence are rarely reported. Here, we present a rare case with parkinsonism and urinary incontinence secondary to DAVF.
\end{abstract}

Case Presentation: A 50-year-old man was admitted for a progressively worsening weakness of his left lower limb over a year with urinary incontinence for four months. Neurological examination revealed rigidity and bradykinesia of the left upper limb without an obvious tremor. Magnetic resonance imaging exhibited diffuse white matter hyperintensities in bilateral centrum semiovale. Cranial magnetic resonance venography revealed extensive tortuous venous malformations. Digital subtraction angiography exhibited multiple bilaterally distributed DAVF in the superior sagittal sinus, supplied by bilateral occipital arteries and superficial temporal arteries. In the one year follow-up, the patient with multiple DAVF bilaterally distributed was completely cured after endovascular treatment according to the symptoms and the follow-up imaging results.

Conclusion: This report enriches the clinical presentations of DAVF with parkinsonism and urinary incontinence, which is a crucial diagnostic clue to be aware of the possibility. Moreover, these rare symptoms can be relieved by timely endovascular treatment.

Keywords: Dural Arteriovenous Fistula (DAVF); Occipital Artery (OA); Superior Sagittal Sinus (SSS); Parkinsonism; Urinary incontinence; Endovascular Treatment (EVT)

\section{Abbreviations}

DAVF: Dural Arteriovenous Fistula; MRI: Magnetic Resonance Imaging; MRV: Magnetic Resonance Venography; DSA: Digital Subtraction Angiography; SSS: Superior Sagittal Sinus; OAs: Occipital Arteries; STAs: Superficial Temporal Arteries; EVT: Endovascular Treatment; CSF: Cerebrospinal Fluid; FLAIR: Fluid Attenuated Inversion Recovery; DWI: Diffusion Weighted Imaging; MRA: Magnetic Resonance Angiography; MRV: Magnetic Resonance Venography; ECA: External Carotid Artery; WML: White Matter Lesion; MMA: Middle Meningeal Artery

\section{Background}

Dural Arteriovenous Fistula (DAVF) is a rare vascular disorder and has been estimated to account for $10 \%$ of all intracranial arteriovenous malformations [1]. Cranial DAVF is characterized by abnormally direct connections between meningeal arteries and meningeal veins or dural venous sinuses [2], which leads feeding arteries to directly drain into cortical veins or a venous sinus [3]. As a result, blood arterialization and increased venous sinus pressure eventually lead to multiple pathological changes including cerebral venous reflux disorders, cerebral edema and vascular rupture. According to the characteristics of venous drainage, DAVF can be classified into three types by Borden-Shucart classification [4]. DAVF has a wide range of locations. The most common location of DAVF is transverse-sigmoid sinus with a slight left-sided predominance and less near the cavernous sinus [5]. It can also occur at tentorial, petrosal, ethmoidal, spinal dura, and superior sagittal sinus locations [6]. The variable clinical manifestations of DAVF depend on not only the anatomical locations but also the hemodynamic properties that is also considered to be the pathological mechanism underlying DAVF formation $[7,8]$. For example, pulsatile tinnitus is a common feature of transverse-sigmoid venous sinuses [9], whereas cranial nerve palsies and visual deterioration are usually due to disorder of cavernous sinus venous system [10]. In addition, DAVF located in the Superior Sagittal Sinus (SSS) and deep venous system mainly
Austin Neurosurg Open Access - Volume 7 Issue 2 - 2021

Submit your Manuscript | www.austinpublishinggroup.com

Yu et al. (C) All rights are reserved
Citation: Yu W, Huang Y, Wu J, Wu J, Li Y and Jin H. Multiple Dural Arteriovenous Fistulas in the Superior Sagittal Sinus Region with Reversible Parkinsonism and Urinary Incontinence as Rare Presentations: A Case Report. Austin Neurosurg Open Access. 2021; 7(2): 1068. 
presents with seizures, Cerebrospinal Fluid (CSF) obstruction, papilledema or dementia [11]. The most aggressive symptoms of DAVF are intracranial hemorrhage and neurological deficits.

Although clinical manifestations of DAVF vary widely, the symptoms of parkinsonism and urinary incontinence are rarely reported. Here, we present a case who suffered from parkinsonism and urinary incontinence secondary to multiple bilaterally distributed DAVF in the SSS region. This case report about rare complications can enrich the clinical presentations affected by venous drainage and help clinicians to avoid misdiagnosis. The written informed consent of the patient was obtained to publish the case details and any accompanying images used in medical journals. And this study was conducted in accordance with the Declaration of Helsinki and the Ethics Committee of Peking University First Hospital.

\section{Case Presentation}

A 50-year-old man was admitted to our hospital for a progressive weakness of his left lower limb over a year along with urinary incontinence for four months. A year ago, the patient suffered from progressive weakness of his left lower limb, accompanied by unstable walking (tends to rush forward and difficulty with stopping when walking). Simultaneously, the patient had suffered from urinary incontinence for four months prior to admission. The patient had a history of hypertension for 10 years and diabetes for 3 years. Additionally, the patient suffered from a traumatic brain injury 25 years ago but it didn't get much attention at that time. Neurologic examinations showed that proximal muscle strength of the left lower extremity was $4 / 5$ and tone of the four extremities was increased without involuntary movement. Bilateral Chaddock's sign were positive.

Cranial Magnetic Resonance Imaging (MRI) on admission exhibited a large lesion in the bilateral parietal and frontal lobes with slightly lower T1 signals, long T2 signals, high signals on T2 Fluid Attenuated Inversion Recovery (FLAIR) and high signals on Diffusion Weighted Imaging (DWI), which was more prominent on the right side (Figure 1A red oval). Cranial MRI with contrast agent on admission suggested that cerebral parenchymal veins in bilateral parietal and frontal lobes were diffusely increased, expansive and tortuous (Figure 2A red square). Head Magnetic Resonance Angiography (MRA) exhibited enlarged bilateral Occipital Arteries (OAs) (Figure 2B red square). Head Magnetic Resonance Venography (MRV) showed multiple obviously expansive and tortuous veins in bilateral frontal and parietal lobes (Figure $2 \mathrm{C}$ red oval) as well as scalp soft tissues (Figure $2 \mathrm{C}$ red arrow). According to these examinations, venous malformations were highly suspected. Therefore, Digital Subtraction Angiography (DSA) was performed, revealing multiple DAVF between External Carotid Artery (ECA) and SSS (Figure 3A red dashed box) with feeding arteries being bilateral OAs (Figure 3A yellow box) and Superficial Temporal Arteries (STAs) (Figure 3A red solid box). Bilateral OAs were enlarged and tortuous (Figure 3A yellow arrow), the right $\mathrm{OA}$ in particular. Simultaneously, a draining vein was obvious within the parietal midline dura, ultimately draining into the front $1 / 2$ of superior sagittal sinus, thus leading to the obvious stenosis of the SSS (Figure 3A red arrow). Extensive tortuous and engorged leptomeningeal veins were observed in the venous phase of the DSA images (Figure $3 \mathrm{~A}$ red oval). Based on these findings, we diagnosed this patient as DAVF with extrapyramidal and urinary incontinence as the first symptoms.

After the diagnosis was finalized, this patient received endovascular embolization. One year after the surgery, the symptoms of parkinsonism and urinary incontinence improved significantly compared to the preoperative conditions. The neurological examination exhibited a substantial improvement over the preoperative evaluation. The lesions in all of the above MRI
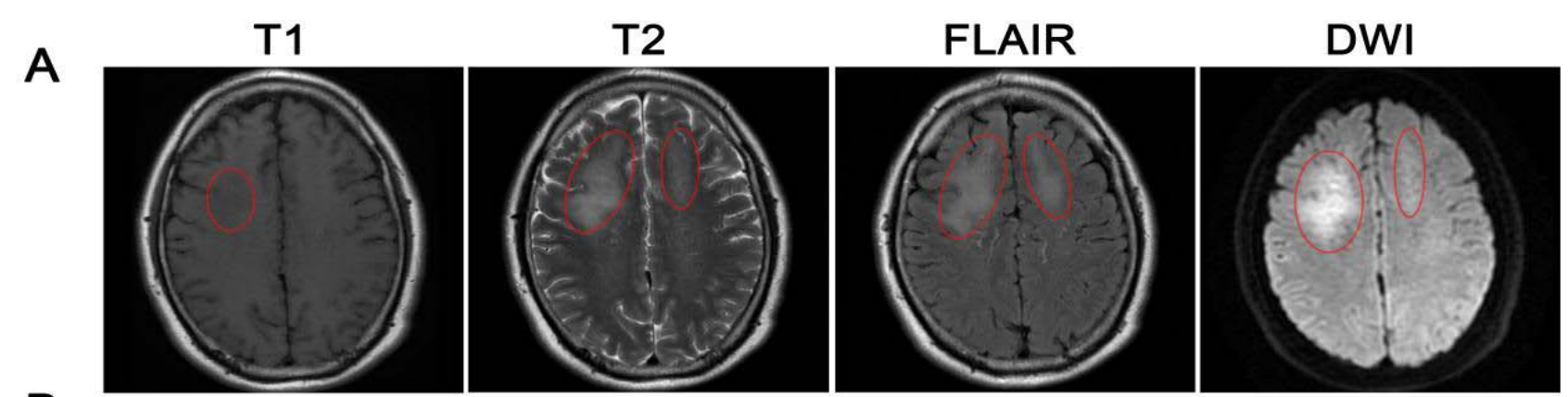

B
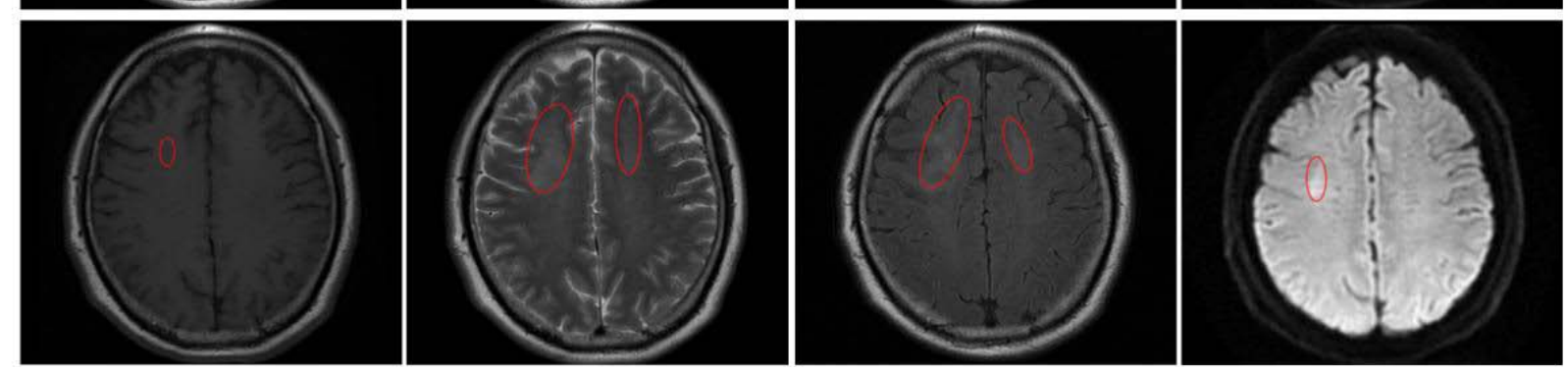

Figure 1: Representative images of multiple cranial MRI sequences. A: T1-weighted, T2-weighted, FLAIR and DWI brain MRI Scans were performed on admission, exhibiting a large slightly lower T1 signal, long T2 signals, a high T2 FLAIR signal and DWI signal in the bilateral parietal and frontal lobes, with the right side more significant (red oval). B: Re-examination of brain MRI scans in Figure 1A one year after the surgical therapy, exhibiting significantly improvement compared to the same scan plane of MRI on admission (red oval). 

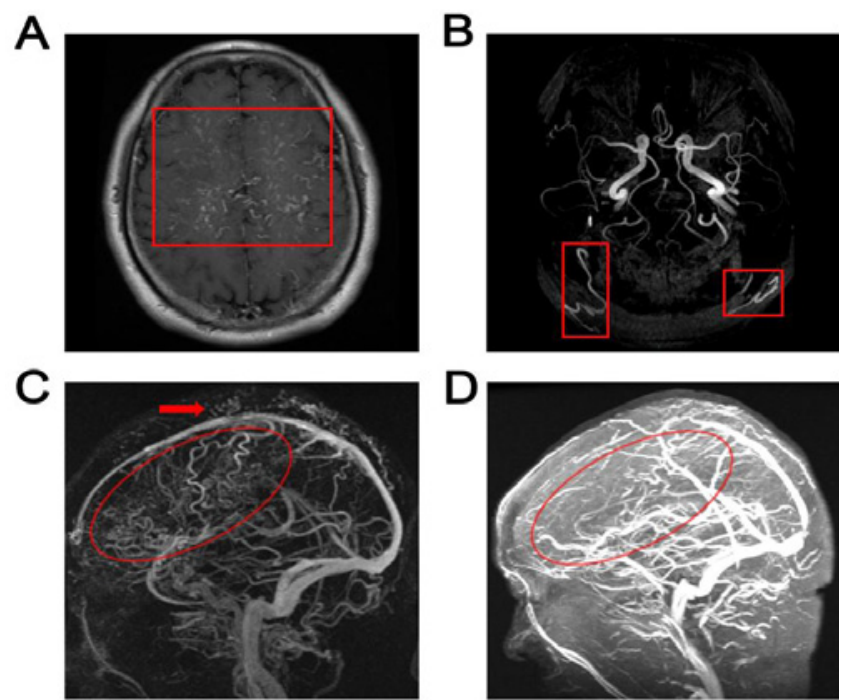

Figure 2: Representative images relative to angiography. A: Cranial enhanced MRI on admission, suggesting cerebral parenchymal veins in bilateral parietal and frontal lobes were diffusely increased, expansive and tortuous (red square). B: MRA on admission exhibited that the bilateral Occipital Arteries (OA) were enlarged, mainly the right OA (red square). C: MRV on admission showed that multiple expansive and tortuous veins were obvious in bilateral frontal and parietal lobes (red oval) and scalp soft tissues (red arrow). D: Re-examination of MRV indicated that expansive and tortuous veins were obviously decreased one year after the surgical treatment (red oval).

sequences were significantly improved on the follow-up MRI (Figure $1 \mathrm{~B}$ red oval), which suggested that these lesions were reversible. Reexamination of MRV indicated that the expansive and tortuous veins were obviously decreased one year later (Figure 2D red oval), which may be the cause of improved diffuse white matter hyperintensities.

\section{Discussion}

Parkinsonism and urinary incontinence are rare manifestations of DAVF which may lead to misdiagnosis and they have been seldom reported [12]. Our study demonstrates a potential relationship between DAVF and parkinsonism as well as urinary incontinence. The following pathophysiological mechanisms are considered to cause parkinsonism in the present case of DAVF. The arteriovenous fistulous developed between the arterial fistula (bilateral occipital and superficial temporal arteries) and the SSS cause a significant arterial steal, which can induce ischemia in frontal, temporal lobes and basal ganglia. And chronic arterial ischemic changes subsequently result in diffuse white matter hyperintensities and basal ganglia damages, ultimately triggering parkinsonism [13]. Although the imaging manifestation of white matter hyperintensities in this case seems similar to that of vascular parkinsonism [14], it is reversible as we described, which cannot be completely explained by chronic arterial ischemia. Similarly, the reversible White Matter Lesion (WML) can also be observed in SSS region in DAVF presenting with dementia [15]. However, there are currently no specific cases of reversible WML in SSS region in DAVF presenting with parkinsonism. Both dementia and parkinsonism secondary to DAVF selectively affect the deep white matter rather than the cortex or the basal ganglia. Thus, venous congestion is more likely to be considered as the mechanism underlying the parkinsonism in DAVF of this case as previously reported [16,17]. Venous congestion leads to relative outflow obstruction, which may cause the hypoperfusion. Subsequently, the patient suffered from progressive urinary incontinence that is even more rare in intracranial DAVF with only approximate five cases reported [12], and the mechanism has not been clearly elaborated. These atypical clinical manifestations of the patient easily lead to misdiagnosis, so neurologists need to keep the reversible etiology in mind. Besides, both symptoms and manifestations in re-examined

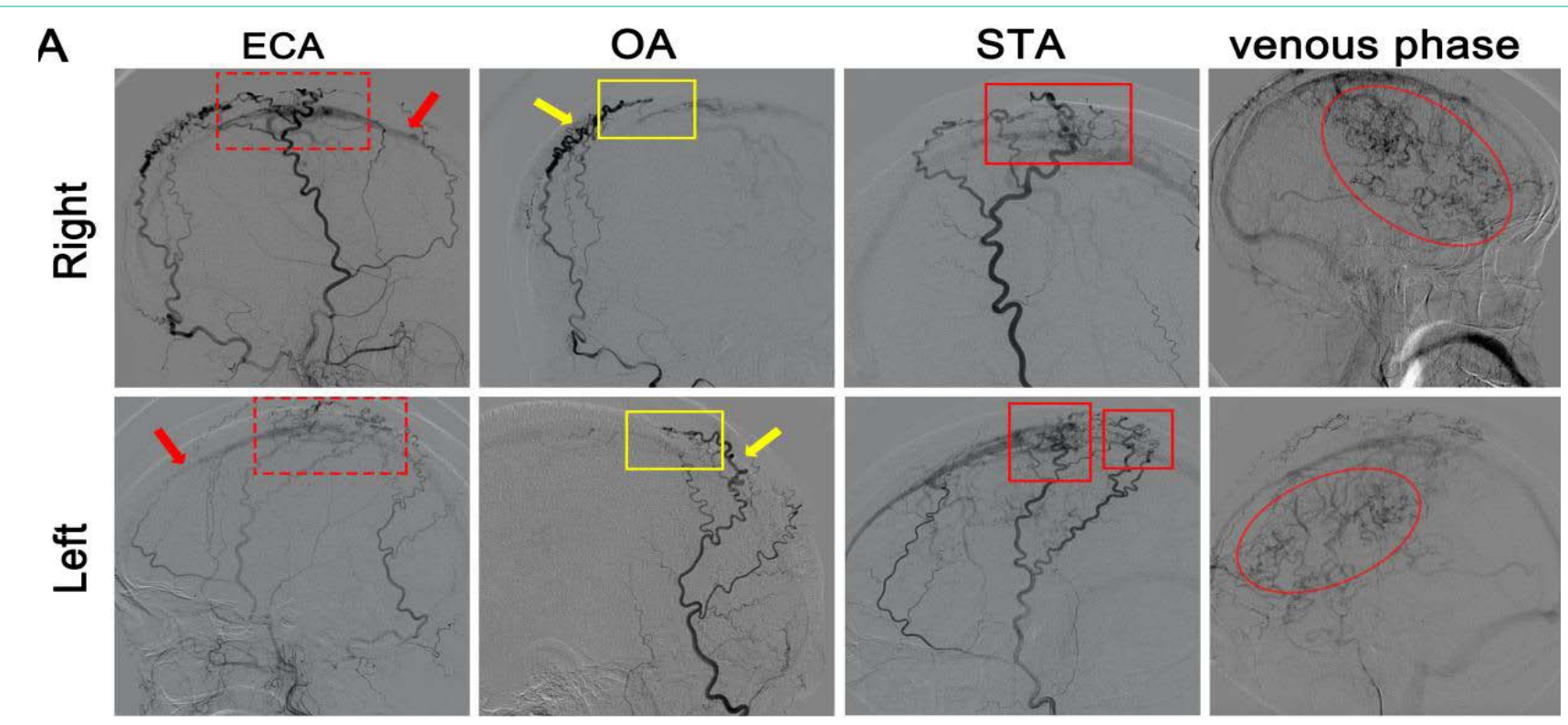

Figure 3: Representative DSA images. A: The DSA images revealed that the branches of external carotid artery (ECA) were served as feeders with venous reflux to the superior sagittal sinus (red dashed box), including Occipital Arteries (OA) (yellow box) and Superficial Temporal Arteries (STA) (red solid box) feeders. Simultaneously, the representative images exhibited that the SSS was obviously stenosis (red arrow) and bilateral OAs were enlarged and tortuous (yellow arrow), especially the right OA. Venous phase in DSA exhibited extensive tortuous expansion of intracranial veins (red oval). 
MRI and MRV of this patient significantly improved one year after the surgical treatment. Therefore, it is probable that earlier treatment for DAVF could have avoided the Parkinsonism and urinary incontinence.

DAVF possesses significant geographic variations with different causes. Most DAVF in the United States and Europe is located in the sigmoid and transverse sinuses, while in Asia it's commonly located in the cavernous sinus [18]. Involvement of SSS region in DAVF is rarely seen [19], which mostly has bilateral feeding arteries from the branches of unilateral or bilateral middle meningeal artery (MMA) [20]. The scalp arteries can also be concerned in DAVF involving SSS region [21]. In addition, OA and STA have also been rarely reported as the feeding arteries, especially the bilateral OAs or STAs [22]. The OA is usually involved in the post-portion of SSS region in DAVF while the STA is involved in the midportion of SSS region. The patient in our study suffered from multiple DAVF involving the SSS regions that are relatively symmetrically distributed. Bilateral OAs or STAs, as the feeding arteries, are rarely reported. Additionally, the scalp arteries are also be involved in the SSS region in DAVF. Currently, sinus thrombosis, trauma, inflammation, and iatrogenic injury are also considered as usual causes [23]. In this case, the patient had a 25year history of trauma on head, which may be the trigger of DAVF's formation. Furthermore, most DAVF involving the SSS region are deemed to demand surgical treatment and Endovascular Treatment (EVT) is considered as the most optimal therapy.

\section{Conclusion}

In summary, DAVF between SSS and bilateral feeding arteries of ECA branches can manifest as parkinsonism, urinary incontinence and bilateral white matter lesions on MRI imaging. This report enriches these rare clinical presentation affected by venous drainage, which is a crucial diagnostic clue to be aware of the possibility. Moreover, although endovascular treatment has been reported to often fail to completely eliminate DAVF in the SSS region, the patient with multiple DAVF bilaterally distributed is completely cured according to the symptoms and the MRI review results. Regrettably, we were unable to obtain the detailed information about the surgical procedure of this patient and the postoperative DSA images were also unavailable. Additionally, more cases and further studies are necessary to validate our results.

\section{Declaration}

Ethics approval and consent to participate: The study was conducted in accordance with the Declaration of Helsinki and the Ethics Committee of Peking University First Hospital. Written informed consent was obtained from the participant in the study.

Consent for publication: Informed consent was obtained from the patient to publish the case details and any images in medical journals.

Availability of data and material: All data generated or analyzed during this study are included in this published article.

Authors' contributions: Yining Huang, Weiwei Yu and Haiqiang Jin were involved in designing the work, analyzing these data. Weiwei $\mathrm{Yu}$ and Haiqiang Jin were the major contributors to writing the manuscript. Qian You was involved in collecting the material of the patient. Haoran Zhang was involved in the analysis of the images. All authors contributed to drafting, revising the paper and giving final approval of the version to be published.

Acknowledgement: We would like to thank the patient in our study for agreeing to publish their available clinical data in medical journals.

\section{References}

1. Kannath SK, Rajendran A, Thomas B, Rajan JE. Volumetric T2-weighted MRI improves the diagnostic accuracy of spinal vascular malformations: comparative analysis with a conventional MR study. J Neurointerv Surg. 2019; 11: 1019-1023.

2. Sorenson T, Giordan E, Cannizzaro D, Lanzino G. Surgical ligation of spinal dural arteriovenous fistula. Acta Neurochir (Wien). 2018; 160: 191-194.

3. Ihn YK, Kim MJ, Shin YS, Kim BS. Dural arteriovenous fistula involving an isolated sinus treated using transarterial onyx embolization. Journal of Korean Neurosurgical Society. 2012; 52: 480-483.

4. Borden JA, Wu JK, Shucart WA. A proposed classification for spinal and cranial dural arteriovenous fistulous malformations and implications for treatment. Journal of neurosurgery. 1995; 82: 166-179.

5. Pabaney AH, Robin AM, Basheer A, Malik G. Surgical Management of Dural Arteriovenous Fistula After Craniotomy: Case Report and Review of Literature. World neurosurgery. 2016; 89: 731.e7-.e11.

6. Gross BA, Du R. Natural history of cerebral arteriovenous malformations: a meta-analysis. Journal of neurosurgery. 2013; 118: 437-443.

7. Reynolds MR, Lanzino G, Zipfel GJ. Intracranial Dural Arteriovenous Fistulae. Stroke. 2017; 48: 1424-1431.

8. Hamada $\mathrm{Y}$, Goto $\mathrm{K}$, Inoue $\mathrm{T}$, Iwaki $\mathrm{T}$, Matsuno $\mathrm{H}$, Suzuki $\mathrm{S}$, et al Histopathological aspects of dural arteriovenous fistulas in the transversesigmoid sinus region in nine patients. Neurosurgery. 1997; 40: 452-456; discussion 6-8.

9. Kim MS, Han DH, Kwon OK, Oh CW, Han MH. Clinical characteristics of dural arteriovenous fistula. Journal of clinical neuroscience: Official journal of the Neurosurgical Society of Australasia. 2002; 9: 147-155.

10. van Dijk JM, terBrugge KG, Willinsky RA, Wallace MC. Clinical course of cranial dural arteriovenous fistulas with long-term persistent cortical venous reflux. Stroke. 2002; 33: 1233-1236.

11. Cognard C, Casasco A, Toevi M, Houdart E, Chiras J, Merland JJ. Dural arteriovenous fistulas as a cause of intracranial hypertension due to impairment of cranial venous outflow. Journal of neurology, neurosurgery, and psychiatry. 1998; 65: 308-316

12. Lai J, Heran MKS, Stoessl AJ, Gooderham PA. Reversible Parkinsonism and Rapidly Progressive Dementia Due to Dural Arteriovenous Fistula: Case Series and Literature Review. Mov Disord Clin Pract. 2017; 4: 607-611.

13. Yamanouchi $H$, Nagura $H$. Neurological signs and frontal white matter lesions in vascular parkinsonism. A clinicopathologic study. Stroke. 1997; 28: 965969.

14. Matsuda S, Waragai M, Shinotoh $\mathrm{H}$, Takahashi $\mathrm{N}$, Takagi $\mathrm{K}$, Hattori $\mathrm{T}$. Intracranial dural arteriovenous fistula (DAVF) presenting progressive dementia and parkinsonism. J Neurol Sci. 1999; 165: 43-47.

15. Labeyrie MA, Lenck S, Saint-Maurice JP, Bresson D, Houdart E. Dural arteriovenous fistulas presenting with reversible dementia are associated with a specific venous drainage. Eur J Neurol. 2014; 21: 545-547.

16. Zeidman SM, Monsein LH, Arosarena O, Aletich V, Biafore JA, Dawson RC et al. Reversibility of white matter changes and dementia after treatment of dural fistulas. AJNR Am J Neuroradiol. 1995; 16: 1080-1083.

17. Nogueira RG, Baccin CE, Rabinov JD, Pryor JC, Buonanno FS, Hirsch JA. Reversible parkinsonism after treatment of dural arteriovenous fistula. J Neuroimaging. 2009; 19: 183-184

18. Satomi J, Satoh K. [Epidemiology and etiology of dural arteriovenous fistula]. 
Brain Nerve. 2008; 60: 883-886

19. AA, SI. Treatment of a superior sagittal sinus dural arteriovenous fistula with Onyx: technical case report. Neurosurgery. 2006; 59: ONSE169-170; discussion ONSE-70.

20. Oh JS, Yoon SM, Shim JJ, Bae HG. Transcranial direct middle meningeal artery puncture for the onyx embolization of dural arteriovenous fistula involving the superior sagittal sinus. J Korean Neurosurg Soc. 2015; 57: 54 57.

21. Watanabe J, Maruya J, Nishimaki K, Ito Y. Onyx removal after embolization of a superior sagittal sinus dural arteriovenous fistula involving scalp artery. Surg Neurol Int. 2016; 7: S410-S414.
22. Hou K, Ji T, Guo Y, Xu B, Xu K, Yu J. Current Status of Endovascular Treatment for Dural Arteriovenous Fistulas in the Superior Sagittal Sinus Region: A Systematic Review of the Literature. World Neurosurg. 2019; 122: 133-143.

23. Gross BA, Du R. The natural history of cerebral dural arteriovenous fistulae. Neurosurgery. 2012; 71: 594-602; discussion-3. 